Weihui Jiang, Peiyao Shen and Ju Gu*

\title{
Nanocrystalline cellulose prepared by double oxidation as reinforcement in polyvinyl alcohol hydrogels
}

https://doi.org/10.1515/polyeng-2019-0258

Received August 19, 2019; accepted October 31, 2019; previously published online December 5, 2019

\begin{abstract}
As a biopolymer with high mechanical strength, nanocellulose was increasingly studied to improve polymer properties. In this study, nanocrystalline cellulose (NCC) was efficiently isolated from eucalyptus pulp by double oxidation (ammonium persulfate oxidation and ultrasonic oxidation). The total yield of NCC $(405.1 \pm 180.5 \mathrm{~nm}$ long and $31.7 \pm 9.5 \mathrm{~nm}$ wide) was $38.3 \%$. A novel hybrid hydrogel was produced from polyvinyl alcohol (PVA) and NCC using the freeze-thaw technique. In this hybrid architecture, hydrogen bonds were formed between PVA and NCC. With the increasing proportion of NCC, the pore size of hydrogels shank gradually and the structure of the hybrid hydrogels became denser. The tensile strength of PVA/NCC hybrid hydrogels increased by $42.4 \%$ compared to the neat PVA hydrogel. The results showed that NCC can improve the swelling, thermal properties, and water evaporation rate of PVA hydrogels due to the hydrophilic hydroxyl groups of NCC and hydrogen bonds between PVA and NCC, indicating that PVA hydrogels would have a wider range of application due to the existence of NCC, a green hybrid filler. Most importantly, this novel double oxidation method for preparing nanocellulose will promote an efficient production of nanocellulose.
\end{abstract}

Keywords: nanocrystalline cellulose; polyvinyl alcohol hydrogels; swelling properties; tensile strength; water evaporation rate.

\section{Introduction}

As a three-dimensional network structure material, hydrogels have been studied extensively in the fields of

*Corresponding author: Ju Gu, School of Materials Science and Engineering, South China University of Technology, 381 Wushan Road, Guangzhou 510640, China, e-mail: psjgu@scut.edu.cn Weihui Jiang and Peiyao Shen: School of Materials Science and Engineering, South China University of Technology, 381 Wushan Road, Guangzhou 510640, China biomedicine, agriculture, and environmental sciences for their excellent chemical and physical properties [1] and used in a variety of applications [2], i.e. soft contact lens, separation membranes, biosensors, artificial muscles, and flow control [3], superabsorbents [4], and drug delivery devices [5, 6], due to their unique properties such as stimulus-responsive properties, sorption, penetrability, and elasticity. Polyvinyl alcohol (PVA), widely used in hydrogel fabrication by freeze-thaw cycles due to its chemical stability, high elasticity, and wear resistance [7], is a polyhydric environment-friendly and nontoxic polymer [8]. To extend the practical application of PVA hydrogels, many efforts have been done to improve the mechanical strength and thermal properties of hydrogels, such as introducing dangly comb-chains [9], adding polymer particles or filler such as nanocellulose and graphene oxide in hydrogel networks [10, 11], cold-treating or freeze-drying [12], incorporating clay with polymer [13], and forming interpenetrating network structure [14]. Besides, the swelling properties of hydrogels are one of the important properties related to applications. For example, they are required to have good swelling properties when hydrogels are used as wound dressings [15]. However, in the context of green environmental protection, finding a green filler to improve the mechanical properties of the hydrogel remains a huge challenge.

Nanocrystalline cellulose (NCC), a natural biopolymer with high crystallinity, is one of the most abundant natural polymers in the world. With the development of nanotechnology, the preparation and application of nanocellulose have become another hot research subject. The methods for preparing nanocellulose mainly include TEMPO oxidation [16] and sulfuric acid hydrolysis [17], but both of them have some toxicity. Various sources, such as rice straws, wood, shells, seed fiber, and waste medium-density fiberboards, have been studied to obtain nanocellulose [18-20]. In the context of green composites, nanocellulose is often regarded as an ideal candidate reinforcement or green filler because of its low cost, low density, renewable resource origin, and biodegradability [21]. In recent years, nanocellulose has been extensively studied in many aspects, such as reinforced polymers due to its high aspect ratio, excellent 
mechanical properties, and thermal stability [22]. It has been reported that natural fiber-reinforced polymer composites with excellent properties have been manufactured, and its tensile moduli and strength are $40 \mathrm{GPa}$ and $280 \mathrm{MPa}$, respectively [23]. Additionally, nanocellulose is nondegradable in the human body, which lacks the enzymes to degrade cellulose, and it has been shown to be biocompatible, which indicates that nanocellulose can be used as a biomedical engineering material [24, 25]. Both nanocellulose and PVA are polyhydroxy polymers, so they have good compatibility. Therefore, it is feasible to choose NCC as a filler for a PVA matrix, and this hybrid hydrogel can be applied as a mask or a wound dressing.

In this work, NCC was prepared by double oxidation, which is a new process for preparing nanocellulose, and a novel hybrid hydrogel was produced from PVA and NCC. Besides, the microstructure and mechanical properties of hydrogels have been studied. The swelling, thermal properties, and water evaporation rate of PVA/NCC hybrid hydrogels also have been investigated.

\section{Materials and methods}

\subsection{Materials}

PVA was provided by Sinopharm Chemical Reagent Co. Hydrogen peroxide $\left(\mathrm{H}_{2} \mathrm{O}_{2}\right.$;30\%; Guangzhou Chemical Co.), sodium hydroxide (NaOH; Tianjin Fuchen Chemical Co.), and ammonium persulfate $\left[\left(\mathrm{NH}_{4}\right)_{2} \mathrm{~S}_{2} \mathrm{O}_{8}\right.$; Shanghai Runjie Chemical Reagent Co.] were used to isolate NCC from eucalyptus pulp.

\subsection{Preparation of NCC}

Eucalyptus pulp (6 g) was immersed into $10 \mathrm{wt} \% \mathrm{NaOH}$ solution $(200 \mathrm{ml})$ for more than $5 \mathrm{~min}$ at room temperature, and then the filtered pulp was oxidized using $20 \mathrm{wt} \%\left(\mathrm{NH}_{4}\right)_{2} \mathrm{~S}_{2} \mathrm{O}_{8}$ solution $(500 \mathrm{ml})$ at $80^{\circ} \mathrm{C}$ for $90 \mathrm{~min}$. Then, the product was centrifuged twice at $4000 \mathrm{rpm}$ for $5 \mathrm{~min}$ and adjusted to alkalinity after the addition of deionized water $(200 \mathrm{ml})$. Finally, the product was further ultrasonically oxidized using $30 \% \mathrm{H}_{2} \mathrm{O}_{2}(10 \mathrm{ml})$ at $70^{\circ} \mathrm{C}$ for $20 \mathrm{~min}$. The reaction came to a halt when $200 \mathrm{ml}$ water was added, and the precipitates were subsequently dialyzed against water using regenerated cellulose dialysis membrane with a molecular weight cutoff of 14,000 for about 6 days. The NCC suspension was obtained after the final product was centrifuged at $4000 \mathrm{rpm}$ for $5 \mathrm{~min}$.

\subsection{Preparation of PVA/NCC hybrid hydrogels}

The PVA/NCC solution was prepared by ultrasonically stirring PVA solution (15 wt\%) with NCC suspension $(0,1,3,5$, and $7 \mathrm{wt} \%$ polymer basis) for $20 \mathrm{~min}$ at room temperature and then transferred into the square mold. After that, the PVA/NCC hybrid hydrogels were obtained by subjecting the solutions to freeze-thaw cycles repeated five times $\left(16 \mathrm{~h}\right.$ at $-20^{\circ} \mathrm{C}$ and $3 \mathrm{~h}$ at $25^{\circ} \mathrm{C}$ ).

\subsection{Characterization}

\subsubsection{Fourier transform infrared spectroscopy (FTIR)}

FTIR spectroscopy was used to examine the chemical structure of NCC and PVA/NCC hybrid hydrogel (PerkinElmer, USA). Dry sample (2 mg) was mixed with $200 \mathrm{mg}$ dry $\mathrm{KBr}$, and the mixture was pressed into a disk for spectrum recording. The spectra were obtained at $4 \mathrm{~cm}^{-1}$ resolution over $4000-450 \mathrm{~cm}^{-1}$ range and averaged from 60 scans in transmittance mode.

\subsubsection{Scanning electron microscopy (SEM)}

The morphology of pure PVA hydrogels and PVA/NCC hybrid hydrogels was obtained by cold field emission SEM (Zeiss Merlin, Germany). For morphological characterization, the hydrogels after swelling in water (to an equilibrium state) were freeze dried at $-52^{\circ} \mathrm{C}$ for $8 \mathrm{~h}$. The samples were immobilized on an aluminum plate with conductive carbon tape and coated with gold at $15 \mathrm{~mA}$ for 2 min under vacuum condition. The samples were imaged at 20-800,000 magnification at 1-2 nA current intensity and $0.5-30 \mathrm{kV}$ accelerating voltage.

\subsubsection{X-ray diffraction (XRD)}

The crystal structure of NCC was studied using XRD (Advance, Bruker) with $\mathrm{CuK} \alpha$ radiation at an operating voltage of $40 \mathrm{kV}$ and a filament current of $40 \mathrm{~mA}$. The samples were grounded to power and spread on a sample stage followed by a slight press with a glass slide. 
The samples were scanned from $4^{\circ}$ to $45^{\circ}(2 \theta)$ at a rate of $1.925^{\circ} / \mathrm{s}$.

\subsubsection{Thermogravimetric analysis (TGA)}

TGA measurements were performed by a Netzsch TG209F1 in a flowing $\mathrm{N}_{2}$ atmosphere with a temperature range from $30^{\circ} \mathrm{C}$ to $600^{\circ} \mathrm{C}$ and a heat rate of $10^{\circ} \mathrm{C} / \mathrm{min}$.

\subsubsection{Mechanical property characterization}

The mechanical properties were tested with a Gotech-AI7000S testing machine according to GB/T1040.32006. The hydrogel samples were cut into dumbbellshaped specimens. The measurements were conducted at a relative humidity of $65 \pm 2 \%$ and temperature of $28 \pm 2^{\circ} \mathrm{C}$. For each sample, at least five specimens were tested, and the results were average values. The crosshead velocity was $10 \mathrm{~mm} / \mathrm{min}$ during normal tensile tests.

\subsubsection{Swelling ratio (SR)}

The swelling behavior of dried hydrogels was determined by immersing the completely dried $\left(60^{\circ} \mathrm{C}\right.$ for $\left.24 \mathrm{~h}\right)$ hydrogel samples in deionized water at room temperature. Swollen gels were weighed by an electronic balance, at predetermined intervals, after wiping the excess surface liquid by filter paper. Before measuring the weight of the swollen samples, the excess surface water was removed by filter papers. The SR was calculated using the following equation [26]:

$$
S R(\%)=\frac{W_{t}-W_{0}}{W_{0}}
$$

where $W_{0}$ is the weight $(\mathrm{g})$ of the dried sample and $W_{t}$ is the weight $(\mathrm{g})$ of the swollen sample at time $t$.

\subsubsection{Water evaporation rate}

The prepared hydrogel after extraction was immersed in deionized water. Taking a certain weight of the hydrogel after it reached swelling equilibrium, the hydrogel was placed in an incubator at $50^{\circ} \mathrm{C}$ for $24 \mathrm{~h}$ in a humid atmosphere of $50 \%$. The weight of the hydrogel was measured at regular intervals until completely dry. The water evaporation rate was calculated using the following equation [15]:

$$
\text { Water lost : } W(\%)=\frac{W_{0}-W_{t}}{W_{0}-W_{d}}
$$

where $W_{0}, W_{t}$, and $W_{d}$ are the starting weight, measured weight, and last weight of the hydrogel, respectively.

\subsubsection{Differential scanning calorimetry (DSC)}

The crystallinity of the hydrogels was studied using a TA Instruments Q2000 DSC with a heating rate of $5^{\circ} \mathrm{C} / \mathrm{min}$ and sample masses of 5-10 mg. The hydrogels were freeze dried before measurement. The crystallinity was calculated using the following equation:

$$
\text { Crystallinity }(\%)=\frac{\Delta H}{W_{(\mathrm{PVA})} \times \Delta H_{m}} \times 100 \%
$$

where $\Delta H$ is the melting enthalpy $(\mathrm{J} / \mathrm{g})$ of the hydrogel, $W_{(\mathrm{PVA})}$ is the mass fraction of PVA in hybrid hydrogel, and $\Delta H_{m}$ is the melting enthalpy of PVA with $100 \%$ crystallinity, and the value of $\Delta H_{m}$ is $138 \mathrm{~J} / \mathrm{g}$ [27].

\section{Results and discussion}

\subsection{Isolation of NCC from eucalyptus pulp}

The FTIR spectrum of eucalyptus pulp and NCC is shown in Figure 1A. The - $\mathrm{OH}$ stretching vibration exhibited a strong absorption peak at about $3300 \mathrm{~cm}^{-1}$, which is a typical cellulose peak [16]. Besides, the peaks at 2900, 1050, and $895 \mathrm{~cm}^{-1}$, which signified stretching vibration of $\mathrm{C}-\mathrm{H}$ and $\mathrm{C}-\mathrm{O}$ bonds and $-\mathrm{C}_{1} \mathrm{H}$ distortion in glycosidic, respectively, are also the typical cellulose peaks. The peak at $1530 \mathrm{~cm}^{-1}$ corresponds to the stretching vibration of the aromatic skeletal in lignin. Besides, two weak peaks at 1247 and $1734 \mathrm{~cm}^{-1}$ are attributed to the $\mathrm{C}-\mathrm{O}$ and $\mathrm{C}=\mathrm{O}$ stretching vibration of hemicelluloses, respectively. The disappearance of peaks at 1530 and $1247 \mathrm{~cm}^{-1}$ indicated thorough removal of lignin and hemicelluloses, which means that nanocellulose was successfully separated from eucalyptus pulp. The peak at $1734 \mathrm{~cm}^{-1}$ has not disappeared, indicating that the hydroxyl group in the nanocellulose was oxidized to a carboxyl group. The total yield of NCC from eucalyptus pulp was about $38.3 \%$. The value is higher than that reported in the literature for nanocellulose obtained by other methods, as shown in Table 1. The morphology of nanocellulose was studied using SEM. A rod-shaped nanocellulose with a large aspect ratio was obtained, as shown 

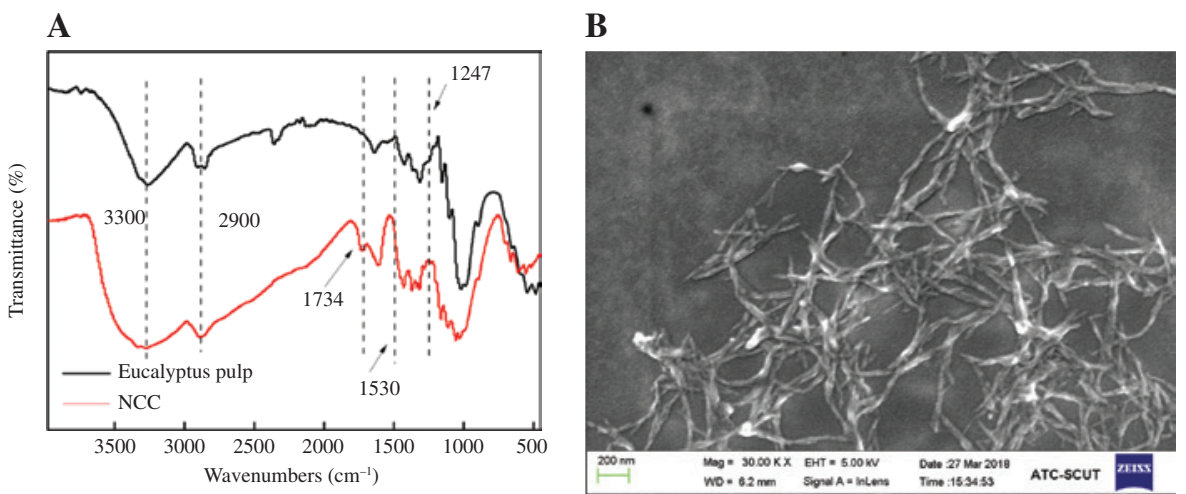

Figure 1: FTIR spectra of eucalyptus pulp and NCC (A) and SEM image of NCC (B).

Table 1: Yield of nanocellulose reported in different literature.

\begin{tabular}{|c|c|c|c|}
\hline Material & Method & Yield (\%) & Ref. \\
\hline Eucalyptus pulp & Double oxidation & 38.3 & This study \\
\hline Tetra Pak packages & Acid hydrolysis & 15.0 & {$[28]$} \\
\hline Microcrystalline cellulose & Acid hydrolysis & $20.6 \pm 5.3$ & [29] \\
\hline Microfibrillated celluloses & Acid hydrolysis & 25 & {$[30]$} \\
\hline
\end{tabular}

in Figure 1B, and it has a length of $405.1 \pm 180.5 \mathrm{~nm}$ and a width of $31.7 \pm 9.5 \mathrm{~nm}$. The NCC suspension can be saved stably for 2 months without significant precipitation, as shown in Figure 2A, which indicates that NCC prepared by double oxidation has good water dispersibility.

Eucalyptus pulp and NCC were analyzed by XRD. In Figure 2B, the XRD diffraction patterns of NCC and eucalyptus pulp used to produce NCC were typical cellulose I as indicated by the three main peaks at $2 \theta=15^{\circ}$, $22.6^{\circ}$, and $34.6^{\circ}$ corresponding to the ( $\left.\begin{array}{lll}1 & -1 & 0\end{array}\right),\left(\begin{array}{lll}2 & 0 & 0\end{array}\right)$, and (0 04 ) lattice planes, respectively [31]. However, the XRD diffraction patterns of NCC also exhibited typical cellulose II characteristic peaks at $2 \theta=12.1^{\circ}$ and $19.8^{\circ}$ corresponding to the $\left(\begin{array}{lll}1 & -1 & 0\end{array}\right)$ and $\left(\begin{array}{lll}1 & 1 & 0\end{array}\right)$ lattice planes, respectively [32]. The above results indicated that eucalyptus pulp contained pure cellulose I, but NCC was a mixture of cellulose I and II. This is because cellulose II may be prepared by $\left(\mathrm{NH}_{4}\right)_{2} \mathrm{~S}_{2} \mathrm{O}_{8}$ oxidation of eucalyptus cellulose I $\left[20 \%\left(\mathrm{NH}_{4}\right)_{2} \mathrm{~S}_{2} \mathrm{O}_{8}, 80^{\circ} \mathrm{C}, 90 \mathrm{~min}\right]$, and a similar phenomenon was reported before [28].

\subsection{Preparation of PVA/NCC hybrid hydrogels}

Figure 3 shows the infrared spectra of NCC and hybrid hydrogels with different NCC contents. The narrow peak at $1647 \mathrm{~cm}^{-1}$ is the deformation vibration peak of the hydroxyl group, and two weak peaks at 2910 and $1456 \mathrm{~cm}^{-1}$ are attributed to the $\mathrm{C}-\mathrm{H}$ stretching vibration and $-\mathrm{CH}_{2}-$ deformation vibration, respectively. The $-\mathrm{OH}$ stretching vibration exhibited a strong absorption peak centered at $3321 \mathrm{~cm}^{-1}$, revealing that significant hydrogen bonds were formed by the freeze-thaw cycles [33]. The C-O stretching vibration peak at $1054 \mathrm{~cm}^{-1}$ was shifted toward the low
A

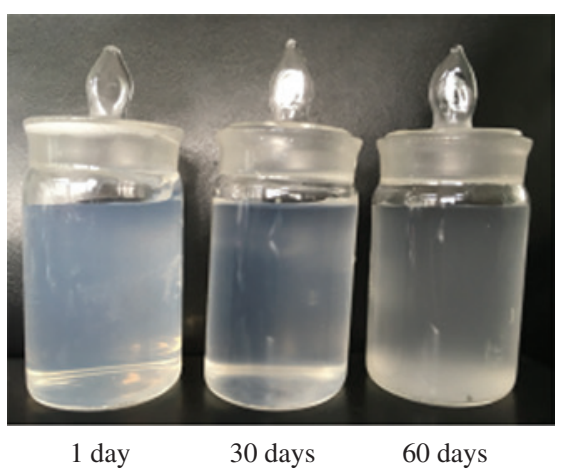

\section{B}

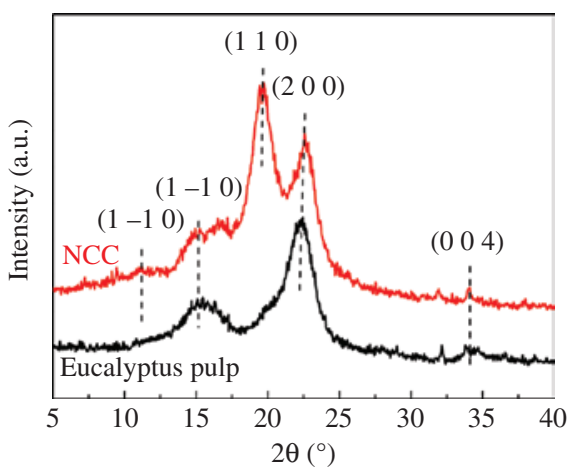

Figure 2: Water dispersion state of NCC suspension $(1 \mathrm{mg} / \mathrm{ml}$ ) at different storage times (A) and XRD profiles of eucalyptus pulp and NCC (B). 


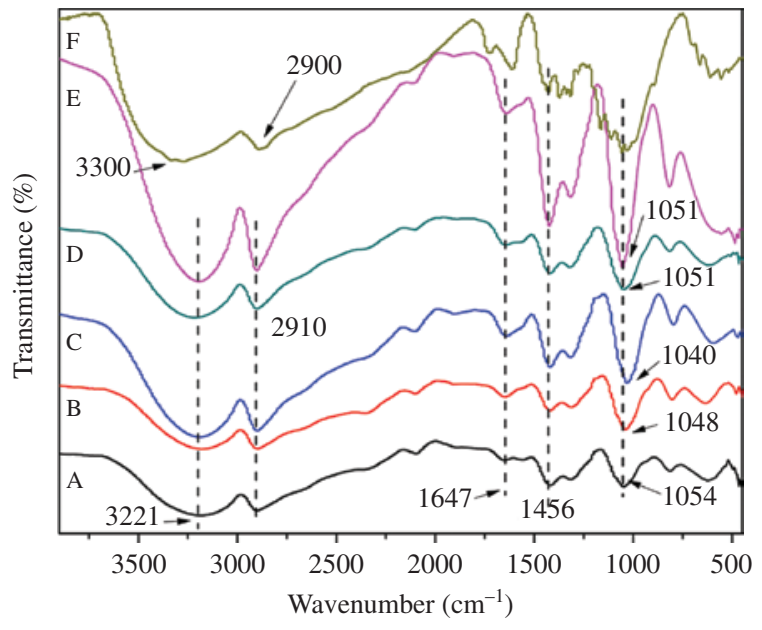

Figure 3: FTIR spectra of PVA hydrogels with (A) 0 wt \%, (B) $1 \mathrm{wt} \%$, (C) $3 w t \%,(D) 5 w t \%,(E) 7 w t \%$ NCC and (F) NCC.

wavenumber, showing that a physical reaction occurred between the polymers of PVA and NCC, and hydrogen bonds were formed between PVA and NCC. When the proportion of NCC in the hydrogel was $3 \mathrm{wt} \%$, the C-O tensile vibration peak at $1054 \mathrm{~cm}^{-1}$ was shifted to $1040 \mathrm{~cm}^{-1}$, indicating that the hydrogen bond formed by the hybrid hydrogel with $3 \mathrm{wt} \%$ NCC was the strongest. It can be seen from the change of the absorption peak at $1054 \mathrm{~cm}^{-1}$ that hybrid hydrogels with $1 \mathrm{wt} \%$ NCC formed more hydrogen bonds than hybrid hydrogels with 5 or $7 \mathrm{wt} \%$ NCC.

The morphological characteristics of the hybrid hydrogels with different NCC contents were studied by SEM. In Figure 4, the pure PVA hydrogels had a porous structure, and the size of pores and the lamellar structures seemed to be affected by the proportion of NCC in the hydrogel. After the addition of NCC, the pore size of the hybrid hydrogel decreases. However, when the proportion of NCC in the hydrogel was $3 \mathrm{wt} \%$ or above, the hydrogels exhibited compact structures. This may be because the crosslinking degree of the hybrid hydrogels increased gradually with the increasing proportion of NCC. In this process, PVA plays the role of a hydrogel scaffold, whereas the NCC acts as a cross-linker due to its strong hydrophilic and hydrogen bonding characteristic.

\subsection{Swelling behavior of PVA/NCC hybrid hydrogels}

Swelling performance is one of the most important properties of hydrogels. Figure 5 shows the swelling-time kinetics curve and swelling equilibrium of different NCC proportions of the hybrid hydrogels. In Figure 5A, different NCC proportions of composite hydrogels have similar swelling processes. In the first $4 \mathrm{~h}$, the swelling rate of the hydrogel gradually increased and slowed down after $5 \mathrm{~h}$. The swelling equilibrium was reached at about $15 \mathrm{~h}$. However, the swelling rate of pure PVA hydrogels is better than hybrid hydrogels, which is attributed to the large pore structure of pure hydrogel. In Figure 5B, the swelling properties of the hybrid hydrogels increased with the increase of the proportion of NCC. The main reason is that NCC was hydrophilic material with polyhydroxyl groups. With the increase of NCC content,
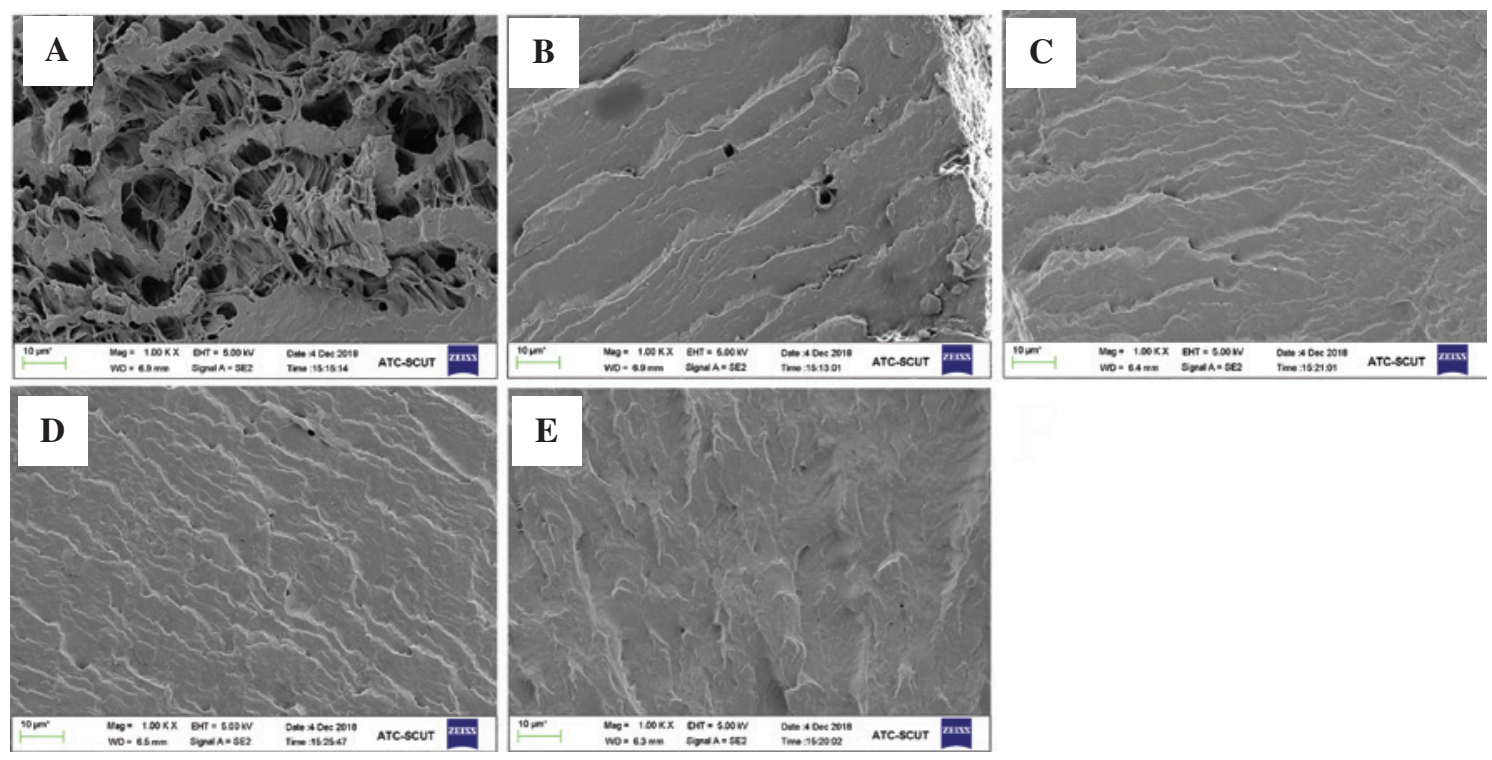

Figure 4: SEM images of PVA hydrogels with (A) 0 wt \%, (B) 1 wt\%, (C) 3 wt \%, (D) 5 wt \%, and (E) 7 wt $\%$ NCC. 
A

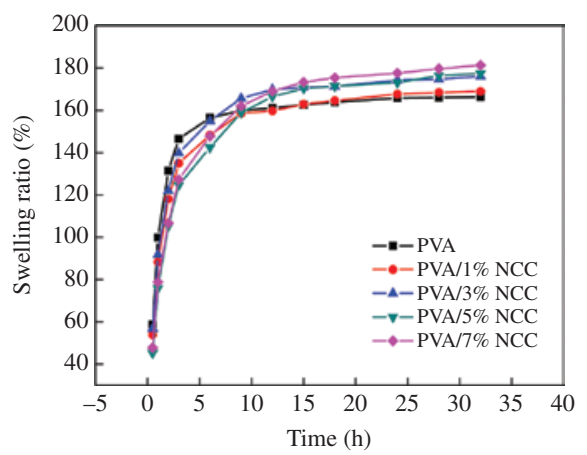

B

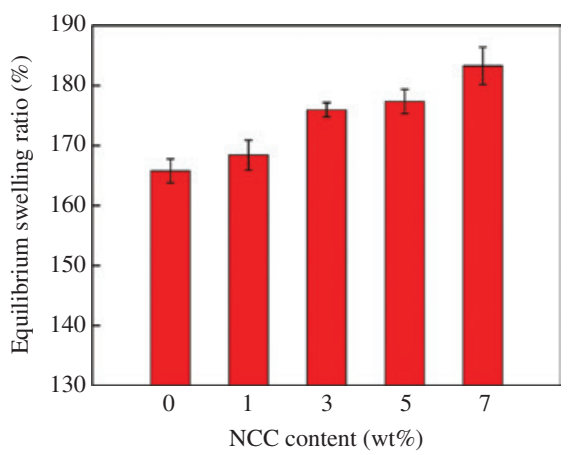

Figure 5: Swelling percentage of hydrogels with different NCC contents (A) and equilibrium SR of hydrogels with different NCC contents (B).

the number of hydrophilic hydroxyl groups increased, which is good for the diffusion of water molecules into the hydrogel, leading to a higher equilibrium swelling ratio [33].

\subsection{Water evaporation rate of PVA/NCC hybrid hydrogels}

In addition to the excellent swelling properties of the hydrogels, the water evaporation rate is also an important characteristic of hydrogels when used as wound dressing [15]. The smaller water evaporation rate of hydrogel dressings could reduce the replacing times for quicker healing, less pain, and great cost savings.

In Figure 6, the percentage of water lost increased sharply in $60 \mathrm{~min}$ between $50 \%$ and $70 \%$. It also reached a constant water loss percentage in $200 \mathrm{~min}$. After $200 \mathrm{~min}$, all of the hydrogels still retained 5\% to $10 \%$ water. This shows that the hydrogels have good water evaporation rate. Besides, the crystallinity of hydrogels was calculated from the melting enthalpy, which was tested by DSC. In Table 2, the addition of NCC increased the crystallinity of PVA hydrogel. In Figure 6, the water evaporation rate of the hybrid hydrogels was better than the pure hydrogels. This is because NCC, a natural biopolymer with high crystallinity, can increase the crystallinity of PVA hydrogels, which can enhance the water stability of the hybrid hydrogels [34]. The water evaporation rate of the hybrid hydrogels decreased with the increase of the proportion of NCC, which was attributed to the number of hydrophilic hydroxyl groups. However, when the proportion of NCC was increased to 5 or $7 \mathrm{wt} \%$, the number of hydrophilic hydroxyl groups was reduced due to the agglomeration of NCC, so the hybrid hydrogels had the best water evaporation rate when the proportion of NCC in the hydrogel was $3 \mathrm{wt} \%$.

\subsection{Mechanical properties of PVA/NCC hybrid hydrogels}

The tensile strengths of pure PVA hydrogels and PVA/ NCC hybrid hydrogels were comparatively evaluated, and the corresponding tensile mechanisms were analyzed. In Figure 7A, the tensile strength of PVA hydrogels was improved with the addition of NCC. When the proportion of NCC in the hydrogel was $3 \mathrm{wt} \%$, the tensile strength of

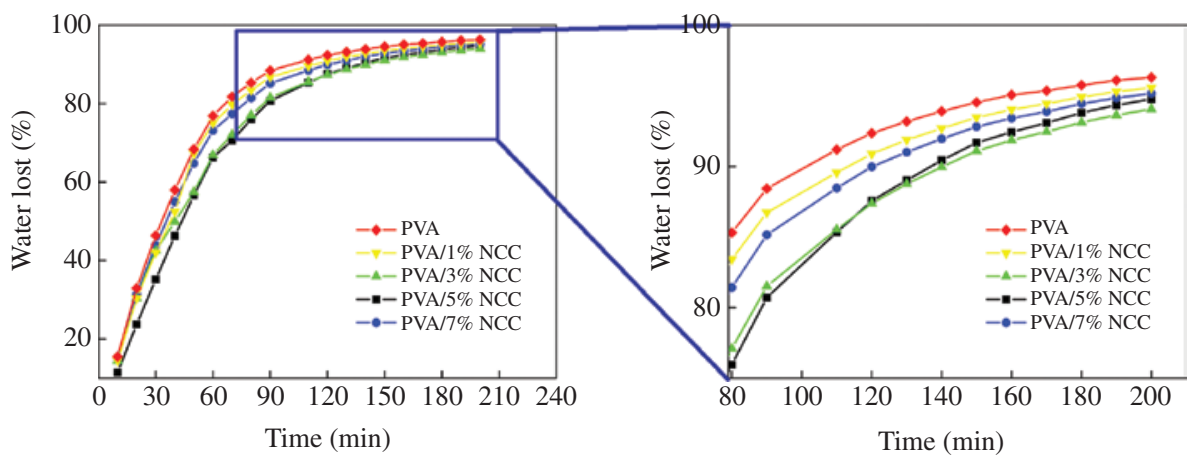

Figure 6: Water loss of the hybrid hydrogels with different NCC contents. 
Table 2: DSC and TGA parameters of PVA/NCC hybrid hydrogels.

\begin{tabular}{lrrrrr}
\hline NCC content & 0 wt\% & 1 wt\% & 3 wt\% & 5 wt\% & 7 wt\% \\
\hline Melting enthalpy $\Delta H(\mathrm{~J} / \mathrm{g})$ & $85.6 \pm 1.2$ & $99.3 \pm 0.6$ & $91.5 \pm 2.5$ & $91.7 \pm 1.4$ & $93.5 \pm 0.7$ \\
Crystallinity $(\%)$ & $61.7 \pm 1.6$ & $72.4 \pm 0.5$ & $68.1 \pm 1.9$ & $69.6 \pm 1.2$ & $72.5 \pm 0.6$ \\
Initial decomposition temperature $\left({ }^{\circ} \mathrm{C}\right)$ & $232.1 \pm 1.1$ & $238.7 \pm 0.8$ & $248.9 \pm 0.6$ & $245.2 \pm 1.2$ & $249.4 \pm 1.1$ \\
\hline
\end{tabular}

$\mathbf{A}$

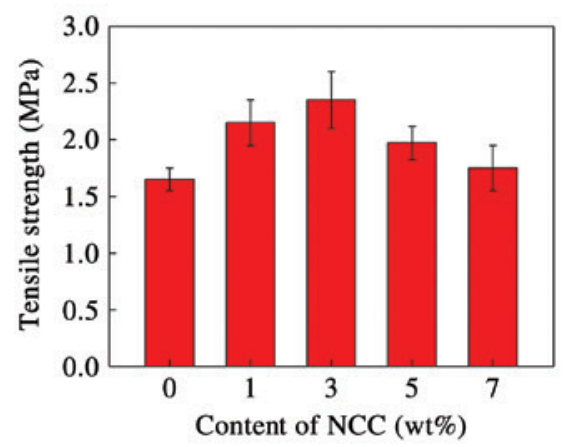

B

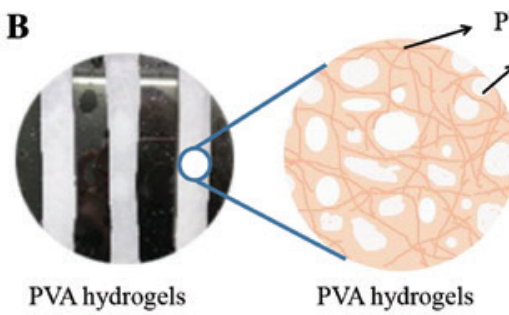

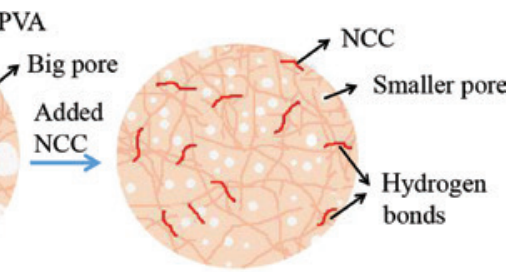

PVA/NCC hydrogels (NCC1 wt\%)

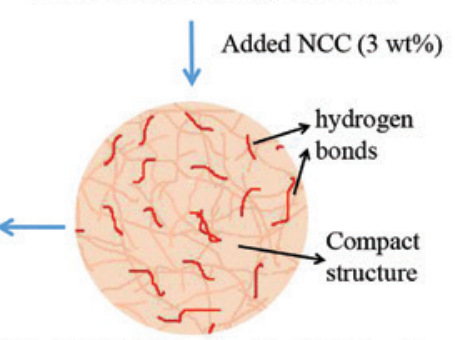

PVA/NCC hydrogels (NCC $>3$ wt\%) PVA/NCC hydrogels (NCC 3 wt\%)

Figure 7: Tensile strength of PVA hydrogels with different NCC contents $(A)$ and proposed model of improved tensile strength of the mechanism (B).

the hybrid hydrogels achieved optimal value among these hydrogels and increased by $42.4 \%$ compared to the pure PVA hydrogel, as the hydrogen bond strengthened gradually and the structure of the hydrogels was more compact with the increasing proportion of NCC. This result was comparable to the results reported for NCC-reinforced hydrogels [35] and even better than the results of some reported NCC-reinforced hydrogels [36]. Therefore, the high strength must be due to the addition of NCC and the remarkable interfacial interactions between PVA chains and NCC [37]. Interestingly, if the proportion of NCC was further increased to 5 or $7 \mathrm{wt} \%$, the tensile strength of the hybrid hydrogel decreased, because NCC may lead to stress concentration due to agglomeration. For better understanding, the structural changes of the PVA/NCC hybrid hydrogel and the proposed nanocellulose-PVA interaction model are shown in Figure 7B.

\subsection{Thermal properties of PVA/NCC hybrid hydrogels}

TGA is a powerful technique to determine the state of polymers and evaluate the intermolecular interaction among polymers in the hydrogel. The initial decomposition temperatures of different hybrid hydrogels are shown in Table 2. In Figure 8 and Table 2, the initial decomposition temperature of hydrogels increased after the addition of NCC, which indicates that the addition of NCC improves the thermal stability of the hybrid hydrogel. The reason is that hydrogen bonds between NCC and PVA increased the combination and strength of the hydrogel [34].

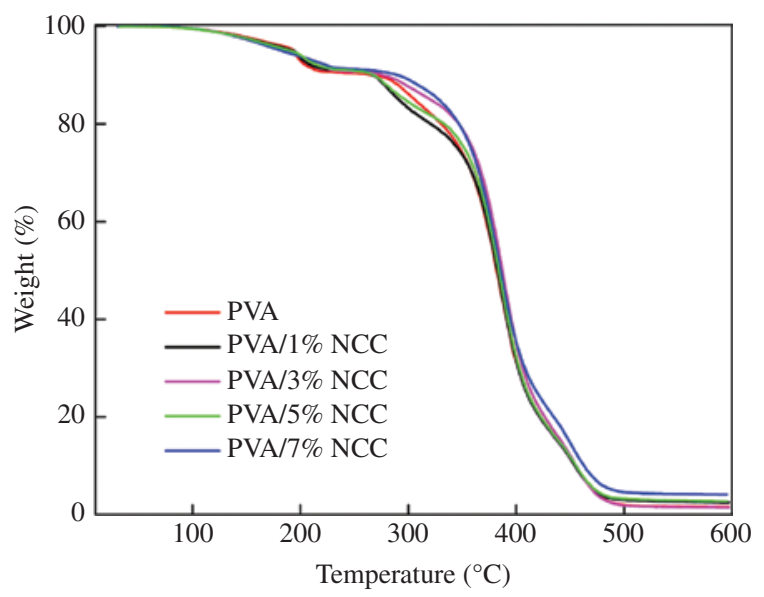

Figure 8: TGA curves of the hybrid hydrogels with different NCC contents. 


\section{Conclusions}

NCC was successfully isolated from eucalyptus pulp by double oxidation. The prepared NCC was a mixture of cellulose I and II and had a length of $405.1 \pm 180.5 \mathrm{~nm}$ and a width of $31.7 \pm 9.5 \mathrm{~nm}$. Then, a novel PVA/NCC hybrid hydrogel was successfully prepared using the freeze-thaw technique. A physical reaction occurred, and hydrogen bonds were formed between the polymers of PVA and NCC. The pure PVA hydrogels had a porous structure, and the size of the pores was smaller gradually and the structure of the hydrogels were more compact with the increasing proportion of NCC. The addition of NCC raised the tensile strength of the hybrid hydrogels, which was attributed to the synergistic effect of hydrogen bonding and compact structure. The tensile strength of the hybrid hydrogels increased by $42.4 \%$ compared to the pure PVA hydrogels. The results showed that NCC can improve the swelling and thermal properties of PVA hydrogels due to the hydrophilic hydroxyl groups of NCC, whereas the water evaporation rate of the hybrid hydrogels is reduced due to hydrogen bonds between NCC and PVA, indicating that NCC as a green hybrid filler enlarges the application fields of PVA hydrogels.

Acknowledgments: The authors are grateful for the financial support from the National Natural Science Foundation of China, Funder Id: http://dx.doi. org/10.13039/501100001809, (grant no. 51173046) and the Guangzhou Municipal Science and Technology Project, China (grant no. 201607010208). The authors also thank Ating Peng for helping with the English.

\section{References}

[1] Yu C, Tang XZ, Liu SW, Yang YL, Shen XC, Gao CC. Int. J. Biol. Macromol. 2018, 117, 1.

[2] Lee WF, Chiang WH. J. Appl. Polym. Sci. 2004, 91, 8.

[3] Kuckling D, Richter A. Polym. Adv. Technol. 2015, 11, 496-505.

[4] Sandeman SR, Gun'Ko VM, Bakalinska OM, Howell CA, Zheng Y, Kartel MT, Phillips GJ, Mikhalovsky SV. J. Colloid Interface Sci. 2011, 358, 582-592.

[5] Liu J, Zhang SM, Chen PP, Cheng L, Zhou W, Tang WX, Chen ZW, Ke CM. J. Mater. Sci. Mater. Med. 2007, 18, 2205.

[6] Wu L, Brazel CS. Int. J. Pharm. 2008, 349, 144-151.

[7] Hassan CM, Peppas NA. J. Appl. Polym. Sci. 2000, 76, 2075-2079.
[8] Pan YS, Xiong DS, Ma RY. J. Central South Univ. Technol. 2006, 13, 27-31.

[9] Kaneko Y, Nakamura S, Sakai K, Aoyagi T, Kikuchi A, Sakurai Y, Okano T. Macromolecules 1998, 31, 6099-6105.

[10] Xu ZY, Li JY. J. Nanosci. Nanotechnol. 2018, 18, 668-675.

[11] Chen J, Shi X, Ren L, Wang Y. Carbon 2017, 111, 18-27.

[12] Pan Y, Ding J, Chen Y, Shen Q. Mater. Process. Rep. 2016, 31, 266-273.

[13] Karimi A, Daud WMAW. Polym. Compos. 2016, 37, 897-906.

[14] Tang Q, Sun X, Li Q, Wu J, Lin J. J. Colloid Int. Sci. 2009, 339, 45-52.

[15] Fan L, Yang H, Yang J, Peng M, Hu J. Carbohydr. Polym. 2016, 146, 427-434.

[16] Gu J, Hsieh YL. ACS Appl. Mater. Interfaces 2015, 7, 4192-4201.

[17] Morán JI, Alvarez VA, Cyras VP, Vázquez A. Cellulose 2008, 15, 149-159.

[18] Jia C, Chen L, Shao Z, Agarwal UP, Hu L, Zhu JY. Cellulose 2017, 24, 2483-2498.

[19] Jiang F, Hsieh YL. Carbohydr. Polym. 2015, 122, 60-68.

[20] Gu J, Hu C, Zhong R, Tu D, Yun H, Zhang W, Leu S-Y. Carbohydr. Polym. 2017, 167, 70-78.

[21] Lee KY, AitomaKi Y, Berglund LA, Oksman K, Bismarck A. Compos. Sci. Technol. 2014, 105, 15-27.

[22] Gu J, Chen W, Lin L, Luo Y, Jia D. Chin. J. Polym. Sci. 2013, 31, 1382-1393.

[23] Oksman K. J. Reinforced Plast. Compos. 2001, 20, 621-627.

[24] Nimeskern L, Martínez Ávila H, Sundberg J, Gatenholm P, Müller R, Stok KS. J. Mech. Behav. Biomed. 2013, 22, 12-21.

[25] Abitbol T, Rivkin A, Cao Y, Nevo Y, Abraham E, Ben-Shalom T, Lapidot S, Shoseyov O. Curr. Opin. Biotechnol. 2016, 39, 76-88.

[26] Dinu MV, Perju MM, DraGan ES. React. Funct. Polym. 2011, 71, 881-890.

[27] Mallapragada SK, Peppas NA. J. Polym. Sci. Pol. Phys. 1996, 34 , 1339-1346.

[28] Xing L, Gu J, Zhang W, Tu D, Hu C. Carbohydr. Polym. 2018, 192, 184-192.

[29] Arrieta MP, Fortunati E, Dominici F, Rayón E, López J, Kenny JM. Carbohydr. Polym. 2014, 107, 16-24.

[30] Goh KY, Ching YC, Chuah CH, Abdullah LC, Liou NS. Cellulose 2016, 23, 379-390.

[31] Henrique MA, Flauzino Neto WP, Silvério HA, Martins DF, Gurge LVA, Barud HDS, de Morais LC, Pasquini D. Ind. Crop. Prod. 2015, 76, 128-140.

[32] Raymond S, Kvick A, Chanzy H. Macromolecules 1995, 28, 8422-8425.

[33] Guan Y, Zhang B, Bian J, Peng F, Sun R. Cellulose 2014, 21, 1709-1721.

[34] Hong H, Liao H, Chen S, Zhang H. Mater. Lett. 2014, 122, 227-229.

[35] Abitbol T, Johnstone T, Quinn TM, Gray DG. Soft Matter 2011, 7, 2373-2379.

[36] Piña-Acosta JA, Zizumbo-Lopez A, Licea-Claverie A. Polym. Compos. 2018, 39, E278-E285.

[37] Fu X, Zhan Y, Meng Y, Li Y, Liao C, Lu Z. Plast. Rubber Compos. 2017, 46, 53-59. 